\title{
A New Hybrid JPEG SyMbol REduCtion IMAge COMPRESSION TECHNIQUE
}

\author{
Bheshaj Kumar ${ }^{1}$,Kavita Thakur $^{2}$ and G. R. Sinha ${ }^{3}$ \\ ${ }^{1,2}$ School of Studies in Electronics, Pt. R. S. University,Raipur,India \\ bheshaj.dewanganerediffmail.com \\ kavithakurarediffmail.com \\ ${ }^{3}$ Shri Shankarachrya College of Engineering \& Technology, Bhilai, India \\ ganeshsinha2003@gmail. com
}

\begin{abstract}
The JPEG standard technique involves three process mapping reduces interpixel redundancy, quantization, which is lossy process and entropy encoding, which is considered lossless process. Lossy JPEG compression is commonly used image compression technique. In the present paper a new hybrid technique has been introduced by combining the JPEG algorithm and Symbol Reduction Huffman technique for achieving more compression ratio. In the symbols reduction method, the number of symbols are reduced by combining together to form a new symbol. As a result of this method the number of Huffman code to be generated also reduced. It is simple, fast and easy to implement. The result shows that the performance of standard JPEG technique can be improved by proposed method. This new hybrid approach achieves about $20 \%$ more compression ratio than the Standard JPEG.
\end{abstract}

\section{KEYWORDS}

Hybrid Image Compression, JPEG, Source Symbol Reduction, Entropy Encoder, Huffman coding.

\section{INTRODUCTION}

Image compression has a prime importance in image communication and storage. In a image compression technique, normally an input image is first manipulated to obtain more compact and/or uncorrelated representation. The manipulated image may be in the form of a mapping, transformation, quantization or a combination of these. Later on the manipulated data is fed to an entropy encoder [1-2]. More emphasis has been given in the recent researches on data manipulation stage, and a growing number of new techniques concerning this stage are introduced in the recent year. There are relatively less innovation in entropy encoding stage. Thus the performance of most image compression scheme can be improved by utilizing an entropy encoder.

An invertible mapping scheme is used in an entropy coder from a sequence of events to a sequence of bits. The aim of entropy coding is the minimization of the number of bits in the bit sequence, while maintaining the concept of invertibility of the mapping [2]. The way of defining the particular events can also be considered a part of entropy coding. The entropy coding can be defined by its own set of intermediate events, based on the input sequence of events as define externally. Entropy coding has been extensively studies in information theory [2-3].

Let $\mathrm{X}=\mathrm{x}_{1} \mathrm{x}_{2} \mathrm{X}_{3} \ldots \ldots \ldots \ldots \ldots \mathrm{x}_{\mathrm{n}}$ be a sequence of independent input symbols, each symbol taking on values from a finite alphabet $A=\left\{a_{1}, a_{2}, \ldots \ldots \ldots a_{m}\right)$. Let $p_{i j}$ denote the probability that $x_{i}$, the $i^{\text {th }}$ symbol in the sequence, takes the value $a_{j}$. Thus the entropy of the sequence is defined as

DOI : 10.5121/ijma.2012.4307 
The International Journal of Multimedia \& Its Applications (IJMA) Vol.4, No.3, June 2012

$$
H(X)=-\sum_{i=1}^{n} \sum_{j=1}^{m} p_{i j} \log _{2} p_{i j}
$$

As per Shannon's source coding theorem [1], the entropy of the sequence is the lower limit on the expected value of the number of bits that is required to encode the sequence [2]. As compared to other algorithms, the Huffman's coding algorithm is generating minimum redundancy codes [3-7]. It has effectively used in text, image, video compression, and conferencing system such as, JPEG, MPEG-2, MPEG-4, and H.263 etc. The Huffman coding scheme collects unique symbols from the source image and calculates its probability value for each symbol and sorts the symbols based on its probability value. Further, two symbols from the lowest probability value to the highest probability value are combined at a time to form a binary tree. Thereafter allocating zero to the left node and one to the right node starting from the root of the tree and finally all zero and one are collected from the root to that particular node in the same order to obtain Huffman code for a particular symbol, [8-11].

There are tremendous amount of work have been done in both lossless and as well as lossy image compression [11-14]. Very limited research work have been reported for Hybrid Image compression [21-27]. A very low bit rate image compression scheme has been proposed by Hsien Wen Tseng and Chin-Chen Chang that combines the good energy-compaction property of DCT with the high compression ratio of VQ-based coding [15]. Lenni Yulianti and Tati R.Mengko have used a hybrid method to improve the performance of Fractal image Compression (FIC) technique by combining FIC (lossy compression) and lossless Huffman coding [16]. S.Parveen Banu and Dr.Y.Venkataramani have proposed a novel hybrid image compression technique for efficient storage and delivery of data based on decomposing the data using daubechies-4 wavelet in combination with the lifting scheme and entropy encoding [17].

In the present venture, for image compression, symbol reduction technique is applied in standard JPEG lossy method. The results provide better compression ratio as compared to JPEG technique.

\section{BACKGROUND OF OUR WORK}

\subsection{Joint Photographic Expert Group (JPEG)}

In most of the images the nearby pixels are associated and therefore comprise redundant information. The primary task is to find less associated representation of the image. Two most important and essential attributes of compression are redundancy and irrelevancy reduction. Redundancy reduction removes repetition from the signal source where as irrelevancy reduction removes parts of the signal that will not be discerned by the signal receiver, namely the Human Visual System (HVS). JPEG stands for Joint Photographic Experts Group. JPEG standard has been established by ISO (International Standards Organization) and IEC (International ElectroTechnical Commission) [18]. The JPEG Image compression system comprises of the following constituents specifically

- Source encoder (DCT based)

- Quantizer

- Entropy encoder [18] 
The International Journal of Multimedia \& Its Applications (IJMA) Vol.4, No.3, June 2012

Figure 1shows the block diagram of a JPEG encoder, which has the following components [18]

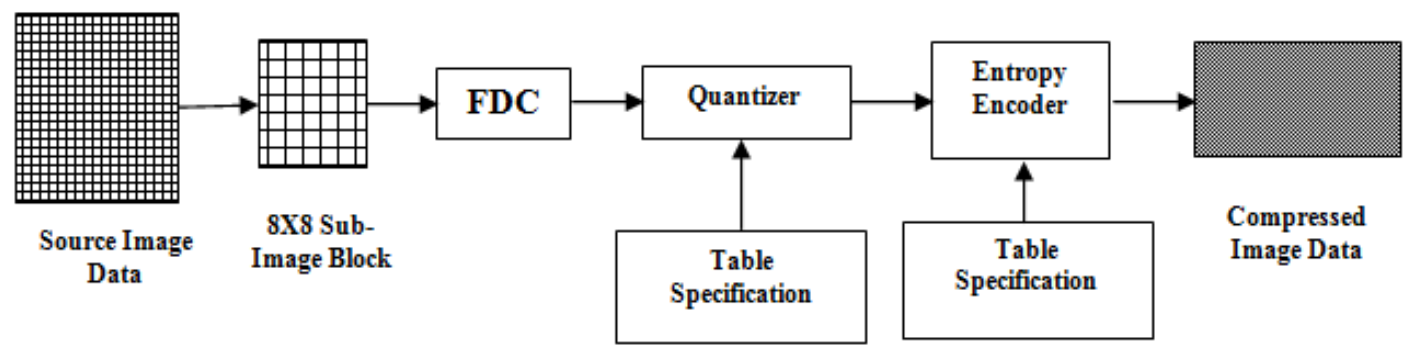

Figure 1. Block Diagram of JPEG Encoder [18]

\subsubsection{Forward Discrete Cosine Transform (FDCT)}

The still images are captured using high precision digital camera and then first partitioned into non-overlapping blocks of size $8 \times 8$ and the image samples are shifted from unsigned integers with range $\left[0\right.$ to $\left.2^{p}-1\right]$ to signed integers with range $\left[-2^{p-1}\right.$ to $\left.2^{p-1}\right]$, where $p$ is the number of bits. It should however be mentioned that to preserve freedom for innovation and customization within implementations, JPEG neither specifies any unique FDCT algorithm, nor any unique IDCT algorithms, because adjacent image pixels are highly correlated [17,18]. The 'forward' DCT (FDCT) processing step lays the foundation for achieving data compression by concentrating most of the signal in the lower spatial frequencies. In principle, the DCT introduces no loss to the source image samples; it merely transforms them to a domain in which they can be more efficiently encoded [19,20]. The 2D Discrete Cosine Transform Pair in two dimensions, for a square matrix, is given by

$$
F(i, j)=\propto(i) \propto(j) \sum_{M=0}^{N-1} \sum_{N=0}^{N-1} f(m, n) \cos \frac{\pi(2 m+1) i}{2 N} \cos \frac{\pi(2 n+1) j}{2 N}
$$

For $\mathrm{i}=0,1,2, \ldots \ldots ., \mathrm{N}-1$ and $\mathrm{j}=0,1,2, \ldots \ldots \ldots \ldots, \mathrm{N}-1$

$$
f(m, n)=\sum_{i=0}^{N-1} \sum_{j=0}^{N-1} \propto(i) \propto(j) F(i, j) \cos \frac{\pi(2 m+1) i}{2 N} \cos \frac{\pi(2 n+1) j}{2 N}
$$

For $\mathrm{m}=0,1,2, \ldots \ldots, \mathrm{N}-1$ and $\mathrm{n}=0,1,2, \ldots \ldots \ldots \ldots, \mathrm{N}-1$

Where,

$$
\propto(k)= \begin{cases}\sqrt{1 / N}, & \text { for } k=0 \\ \sqrt{2 / N}, & \text { for } k=1,2, \ldots ., N-1\end{cases}
$$

\subsubsection{Quantization}

In the next step after FDCT, each of the 64 DCT coefficients block is uniformly quantized according to a quantization table. Each step-size should be chosen as the perceptual threshold or for just noticeable distortion without visual artifacts. Psycho-visual experiments generate a set of quantization tables and these appear in ISO-JPEG standard as a matter of information, but not as a requirement [16-18]. The quantized values are then multiplied at the decoder by the corresponding QT elements to recover the original un-quantized values. All of the quantized 
The International Journal of Multimedia \& Its Applications (IJMA) Vol.4, No.3, June 2012

coefficients are ordered into the zigzag sequence after quantization. This procedure helps to facilitate entropy encoding by placing low-frequency non-zero coefficients before high-frequency coefficients. The DC coefficient, which contains a significant fraction of the total image energy, is differentially encoded $[19,20]$.

\subsubsection{Entropy Coder}

In the entropy encoder ,the final processing step of the JPEG encoder, entropy coding (EC) is achieved by additional lossless compression by encoding the quantized DCT coefficients more compactly based on their statistical characteristics. There are two entropy coding methods Huffman and arithmetic coding specified by the JPEG standard .The baseline sequential JPEG uses Huffman only, but codes with both methods are specified for the other modes of operation. In Huffman coding one or more sets of coding tables are specified by the application. The same table is used to decompress it. The baseline JPEG needs only two sets of Huffman tables - one for DC and the other for AC. Block diagram of the JPEG decoder is shown in Figure no. 2. JPEG decoder performs the inverse operation of the JPEG encoder [18].

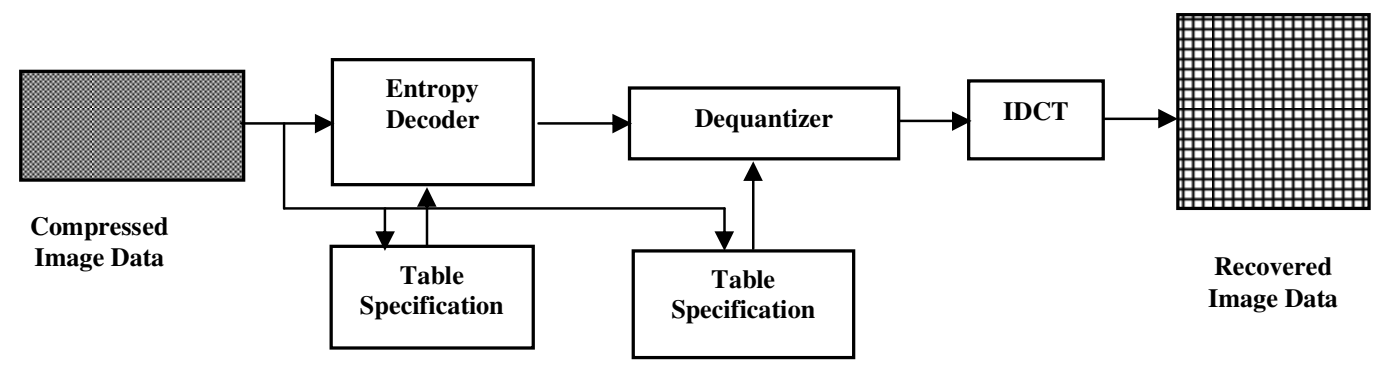

Figure 2. Block Diagram of JPEG Decoder

The use of uniformly sized blocks simplified the compression system, but it does not consider the irregular shapes within the real images [20]. Degradation occurs and it depends on the block size. It is known as blocking effect. A larger block requires more computational power but at the same time it leads to more efficient coding. Image distortion is less annoying for small than for large DCT blocks. Therefore more existing systems use blocks of $8 \times 8$ or 16x16 pixels as a compromise between coding efficiency and image quality [28-29].

\subsection{Symbol Reduction Method}

The number of source symbols plays a very important role in achieving a good compression ratio. A new compression technique has been introduced with a aim to reduce the number of source symbols. The source symbols combined together in the same order from left to right to form a less number of new source symbols. The source symbols reduction explained with an example as shown below. The following eight symbols are assumed as part of an image, A, B, C, D, E, F, G, $\mathrm{H}$. By applying source symbols reduction from left to right in the same sequence, four symbols are combined together to form a new element, thus two symbols ABCD and EFGH are obtained[8]. This technique helps to reduce 8 numbers of source symbols to 2 numbers i.e. $2^{\mathrm{n}}$ symbols are reduced to $2^{(\mathrm{n}-2)}$ symbols. For the first case, there are eight symbols and the respective Symbols and Huffman Codes are A-0, B-10, C-110, D-1110, E-11110, F-111110, G1111110, H-1111111. The proposed technique reduced the eight symbols to two and the reduced Symbols and Huffman codes are ABCD-0, EFGH-1. 
The International Journal of Multimedia \& Its Applications (IJMA) Vol.4, No.3, June 2012

The average number $L_{\text {avg }}$ of bits required to represent a symbol is defined as,

$$
L_{\text {avg }}=\sum_{k=1}^{l} l\left(r_{k}\right) p\left(r_{k}\right)
$$

Where, $r_{k}$ is the discrete random variable for $k=1,2, \ldots \mathrm{L}$ with associated probabilities $\mathrm{p}_{\mathrm{r}}\left(\mathrm{r}_{\mathrm{k}}\right)$. The $1\left(r_{k}\right)$ represents the number of bits used to represent each value of $r_{k}$ [9]. The number of bits required to represent an image is calculated by number of symbols multiplied by $L_{\text {avg }}$ [1-2].In the Huffman coding, probability of each symbols is 0.125 and $L_{\text {avg }}=4.375$.In the proposed technique, probability of each symbol is 0.5 and $L_{\text {avg }}=1.0$. The $L_{\text {avg }}$ confirms that the proposed technique achieves better compression than the Huffman Coding. 8 rows and 8 columns of eight bits greyscale image having 64 symbols are used to calculate required storage size. These two techniques make difference in the coding stage [9]. In the first case, 64 Huffman code are generated by the 64 symbols, whereas the proposed technique generates 16 Huffman codes and reduces $L_{\text {avg }}$. Therefore, the experimental outputs confirm that the source symbols reduction technique helps to achieve more compression ratio [9].

Since the source images firstly divided in $8 \times 8$ sub-block, will have totally 64 symbols. The images are 8 bit grey-scale and the symbol values range from 0 to 255 . To represent each symbol eight bit is required [9]. Therefore, size of an image becomes 64 x $8=512$ bit.

\section{PROPOSED JPEG IMAGE COMPRESSION METHOD}

The image is subdivided into non-overlapping 8x8 sub-image blocks and DCT coefficients are computed for each block. The quantization is performed conferring to quantization table. The quantized values are then rearranged according to zigzag arrangement. After getting zigzag coefficients the remaining coefficients are compressed by the proposed entropy encoder. The block diagram of our proposed method is shown in figure 3 .

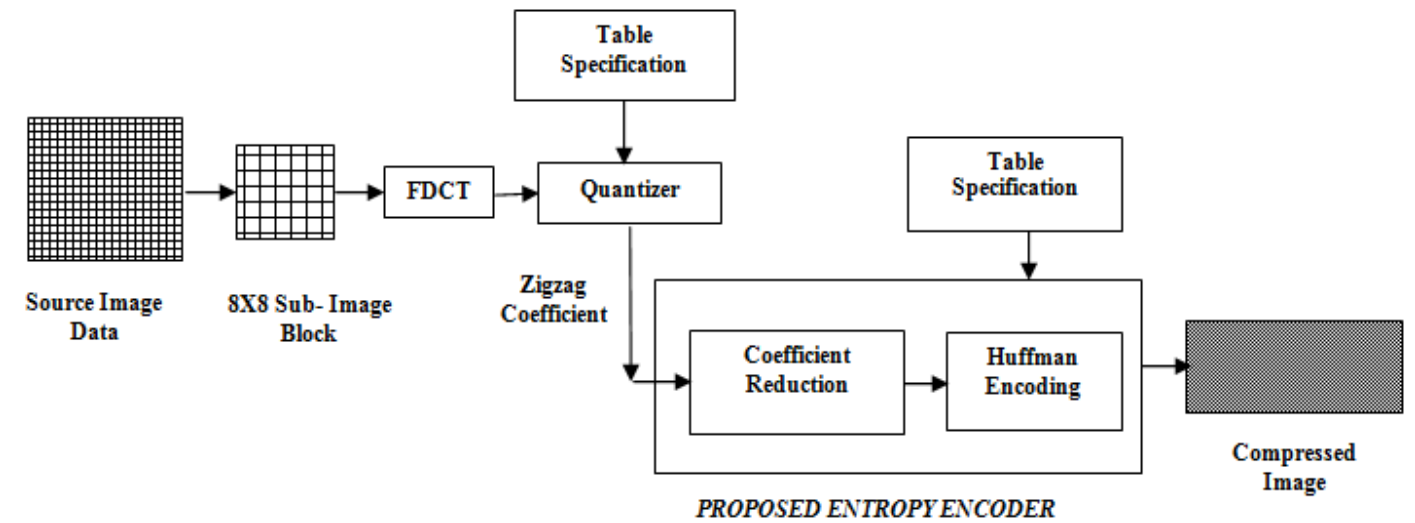

Figure 3. Block Diagram of Proposed JPEG Encoder

\subsection{Algorithm}

1. Input the image data to be compressed.

1. Divide the image into non-overlapping $8 \times 8$ sub images blocks.

2. Shift the gray-levels in the range between $[-128,127]$.

3. Apply DCT on the each sub-image.

4. Quantize the coefficients and the less significant coefficients are set to zero. 
The International Journal of Multimedia \& Its Applications (IJMA) Vol.4, No.3, June 2012

5. Order the coefficients using zigzag ordering and the coefficients obtained are in order of increasing frequency.

6. Compress remaining quantized values by applying proposed entropy encoder.

To reconstruct the image, reverse process of our proposed algorithm are carried out.

As an example, let us consider the $8 \times 8$ block of pixel value component samples shown in Table 1. This block is extracted from one of the images used in the experiments. Table 2 shows the corresponding coefficients obtained by applying the DCT transform to the block in Table 1.

Table 1. An example of pixel values of 8 X8 image block

\begin{tabular}{|c|c|c|c|c|c|c|c|}
\hline 58 & 45 & 29 & 27 & 24 & 19 & 17 & 20 \\
\hline 62 & 52 & 42 & 41 & 38 & 30 & 22 & 18 \\
\hline 48 & 47 & 49 & 44 & 40 & 36 & 31 & 25 \\
\hline 59 & 78 & 49 & 32 & 28 & 31 & 31 & 31 \\
\hline 98 & 138 & 116 & 78 & 39 & 24 & 25 & 27 \\
\hline 115 & 160 & 143 & 97 & 48 & 27 & 24 & 21 \\
\hline 99 & 137 & 127 & 84 & 42 & 25 & 24 & 20 \\
\hline 74 & 95 & 82 & 67 & 40 & 25 & 25 & 19 \\
\hline
\end{tabular}

Table 2. DCT coefficients corresponding to the 8 X8 block in Table 1

\begin{tabular}{|c|c|c|c|c|c|c|c|}
\hline 421.00 & 203.33 & 10.65 & -45.19 & -30.25 & -13.83 & -14.15 & -7.33 \\
\hline-107.82 & -93.43 & 10.09 & 49.21 & 27.72 & 5.88 & 8.33 & 3.28 \\
\hline-41.83 & -20.47 & -6.16 & 15.53 & 16.65 & 9.09 & 3.28 & 2.52 \\
\hline 55.94 & 68.58 & 7.01 & -25.38 & -9.81 & -4.75 & -2.36 & -2.12 \\
\hline-33.50 & -21.10 & 16.70 & 8.12 & 3.25 & -4.25 & -4.75 & -3.39 \\
\hline-15.74 & -13.60 & 8.12 & 2.42 & -3.98 & -2.12 & 1.22 & 0.73 \\
\hline 0.28 & -5.37 & -6.47 & -0.58 & 2.30 & 3.07 & 0.91 & 0.63 \\
\hline 7.78 & 4.95 & -6.39 & -9.03 & -0.34 & 3.44 & 2.57 & 1.93 \\
\hline
\end{tabular}

Now we apply the quantization processes to the DCT coefficients in Table 2. To compute the quantized values, we adopt the JPEG standard default quantization table as shown in Table 3. Table 4 reports the quantized DCT coefficients.

Table 3. Default Quantization Table

\begin{tabular}{|c|c|c|c|c|c|c|c|}
\hline 16 & 11 & 10 & 16 & 24 & 40 & 51 & 61 \\
\hline 12 & 12 & 14 & 19 & 26 & 58 & 60 & 55 \\
\hline 14 & 13 & 16 & 24 & 40 & 57 & 69 & 56 \\
\hline 14 & 17 & 22 & 29 & 51 & 87 & 80 & 62 \\
\hline 18 & 22 & 37 & 56 & 68 & 109 & 103 & 77 \\
\hline 24 & 35 & 55 & 64 & 81 & 104 & 113 & 92 \\
\hline 49 & 64 & 78 & 87 & 103 & 121 & 120 & 101 \\
\hline 72 & 92 & 95 & 98 & 112 & 100 & 103 & 99 \\
\hline
\end{tabular}


The International Journal of Multimedia \& Its Applications (IJMA) Vol.4, No.3, June 2012

Table 4. The quantized DCT coefficients corresponding to the block in Table 1

\begin{tabular}{|c|c|c|c|c|c|c|c|}
\hline 26 & 18 & 1 & -3 & -1 & 0 & 0 & 0 \\
\hline-9 & -8 & 1 & 3 & 1 & 0 & 0 & 0 \\
\hline-3 & -2 & 0 & 1 & 0 & 0 & 0 & 0 \\
\hline 4 & 4 & 0 & -1 & 0 & 0 & 0 & 0 \\
\hline-2 & -1 & 0 & 0 & 0 & 0 & 0 & 0 \\
\hline-1 & 0 & 0 & 0 & 0 & 0 & 0 & 0 \\
\hline 0 & 0 & 0 & 0 & 0 & 0 & 0 & 0 \\
\hline 0 & 0 & 0 & 0 & 0 & 0 & 0 & 0 \\
\hline
\end{tabular}

After quantization, we can observe that there usually are few nonzero and several zero-valued DCT coefficients. Thus, the objective of entropy coding is to losslessly compact the quantized DCT coefficients exploiting their statistical characteristics. The quantized 64 coefficients are formatted by using the zigzag scan preparation for entropy coding.

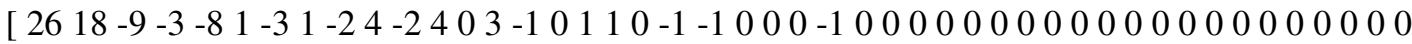

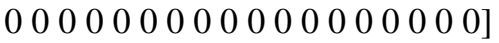

Since many of the coefficients are negative and zero after quantization, each coefficient is checked for either non-zero or zero coefficient, if coefficient is nonzero, add 128 to the coefficient to make a positive integer.

[154 1461191251201291251281261321261320131127012912901271270001270 $00000000000000000000000000000000000000000000]$

Now according to proposed reduction method, from left to right in the same sequence, four symbols are combined together to form a new string, thus these 64 symbols are reduced in 16 symbols.

[(154146119125) (120129125128) (126132126132) (01311270) (1291290127) (127000)

(127000) (0000) (0000) (0000) (0000) (0000) (0000) (0000) (0000) (0000)]

This string is encoded using a predetermined Huffman coding process and finally, it generates compressed JPEG image data.

\section{EVALUATION OF PROPOSED METHOD}

In carrying out the evaluation as part of this research paper, numerical evaluation is adopted. In the case of lossy compression, however, the reproduction is only an approximation to the original image. Measurement of quality and Compression Ratio are a central issue with lossy compression. Since our main target were to reduce the source symbol after quantization, which is totally reversible process, therefore the compression error not considered. So the picture quality (PSNR) values are common to both Standard JPEG method and Proposed JPEG compression method. So in this paper, we only evaluated the compression ratio $\left(C_{R}\right)$ using equation 5 .

$$
\mathrm{C}_{R}=\frac{\text { Original Image Size }}{\text { Compressed Image Size }}
$$


The International Journal of Multimedia \& Its Applications (IJMA) Vol.4, No.3, June 2012

\section{RESULT AND DISCUSSION}

For evaluating the performance of the proposed algorithms, we used 256x256 gray scale versions of the well-known Cameraman, Rice, Coin and Tree images, shown in Figure 4. A software algorithm has been developed and implemented to compress the given image using JPEG standard and Huffman coding techniques in a MATLAB platform.
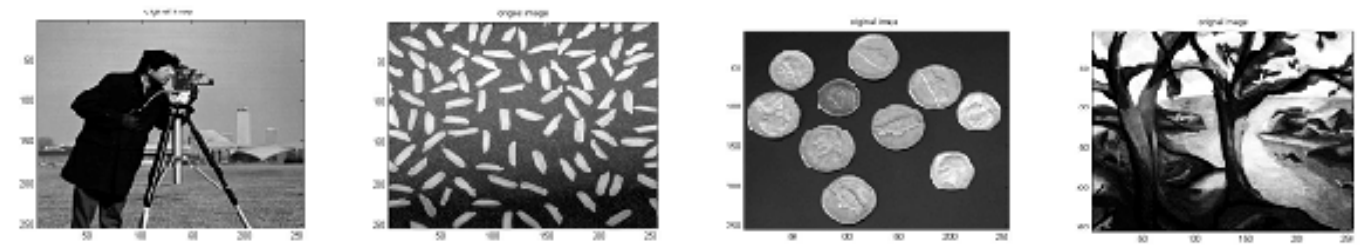

Figure 4. Test images for evaluating the performance of proposed method

Intermediate Images of Cameraman during processing are shown below

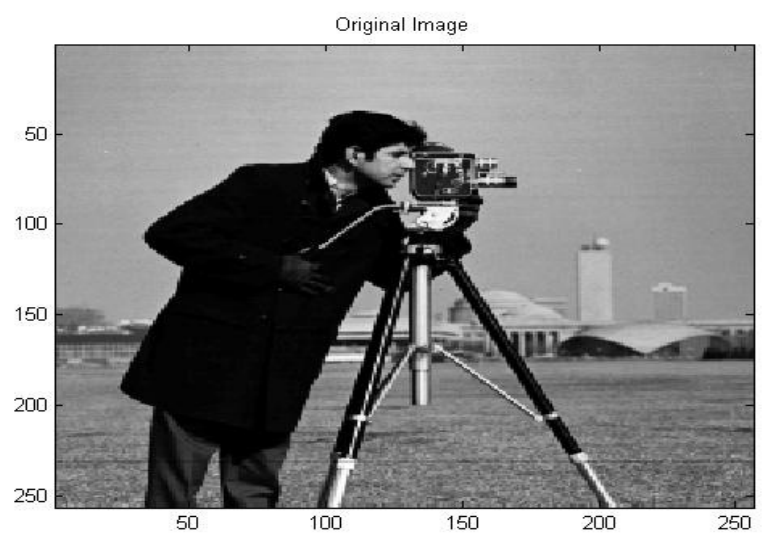

Figure 5. Original Test images

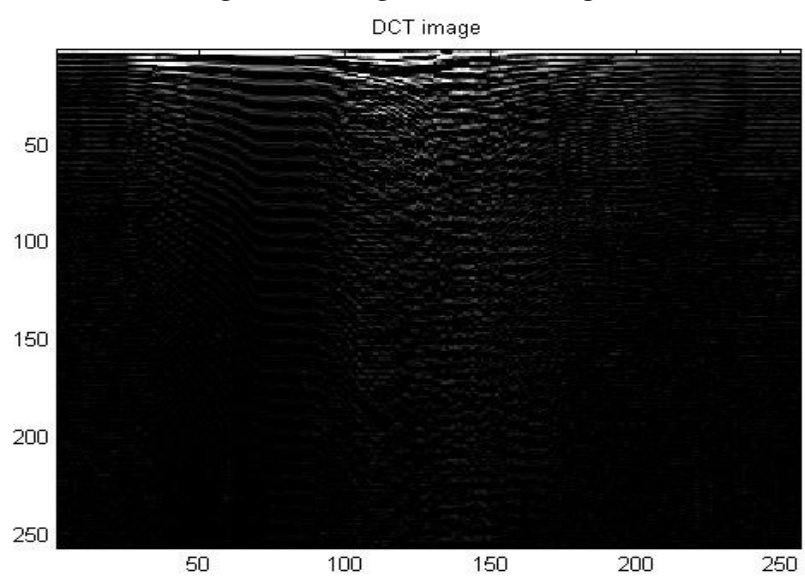

Figure 6. After applying DCT 
The International Journal of Multimedia \& Its Applications (IJMA) Vol.4, No.3, June 2012

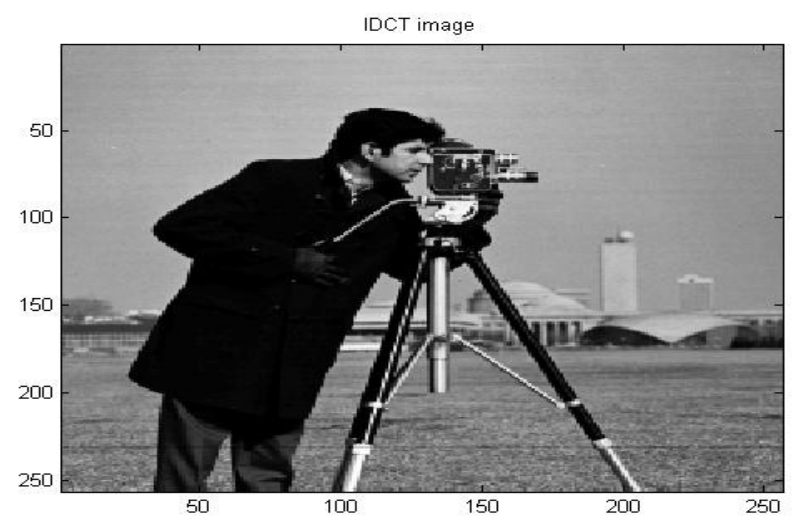

Figure 7. After applying IDCT

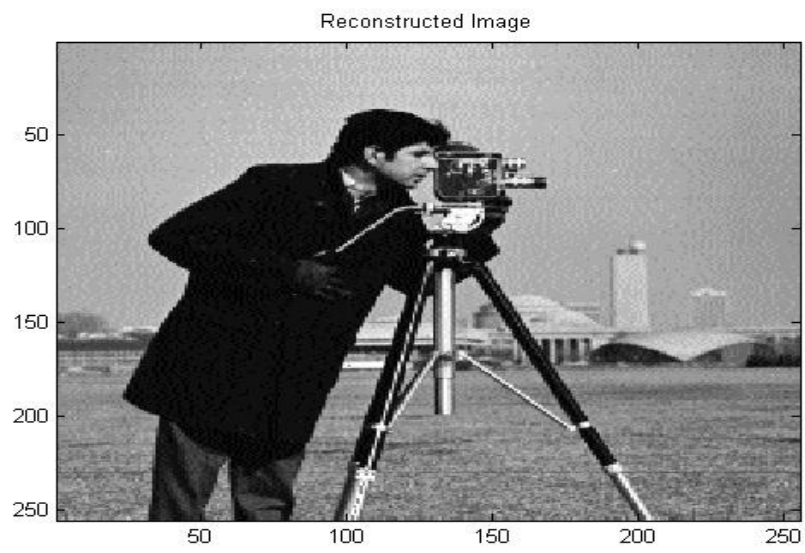

Figure 8. Reconstructed image

The results obtained from the implementation of the proposed algorithm are shown in the table 5 . The compressed ratio of the four test images are calculated and presented in table 5.

Table 5. Compression Ratio of Standard JPEG and Proposed Method

\begin{tabular}{|l|c|c|}
\hline Test Images & $\begin{array}{c}\text { JPEG } \\
\text { Method }\end{array}$ & $\begin{array}{c}\text { Proposed } \\
\text { Method }\end{array}$ \\
\hline Cameraman & 4.13 & 4.94 \\
\hline Rice & 5.68 & 6.45 \\
\hline Coin & 3.84 & 4.73 \\
\hline Tree & 2.53 & 3.08 \\
\hline
\end{tabular}

The result shows that for all test images the compressed size obtained from the proposed technique better than the Standard JPEG method. The proposed compression technique achieves better compression. The results obtained from the present analysis are shown in figure 5 . 
The International Journal of Multimedia \& Its Applications (IJMA) Vol.4, No.3, June 2012

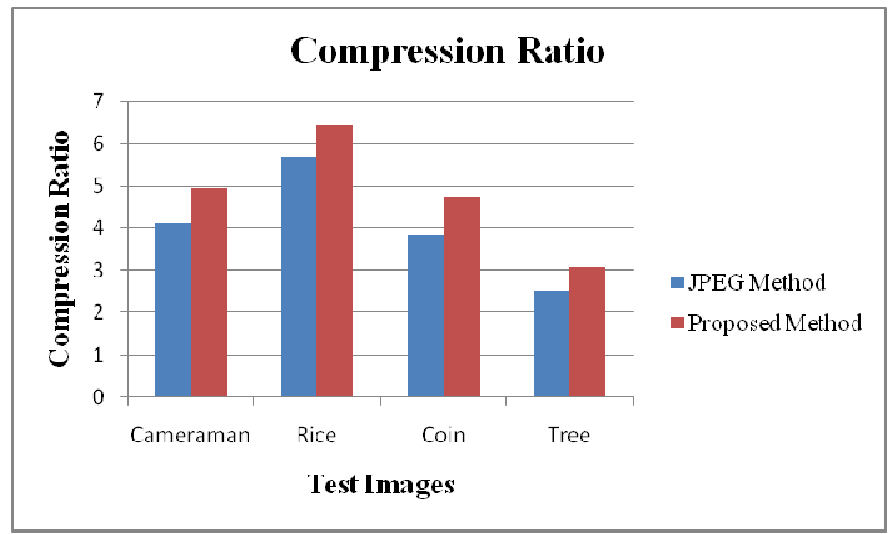

Figure 9. Compression Ratio of Standard JPEG and Proposed Method

Since it is found that the two compression techniques are lossless compression technique, therefore the compression error not considered. So the picture quality (PSNR) values are common to both Standard JPEG method and Proposed JPEG compression method.

\section{CONCLUSION}

The experimentation in present paper reveals that the compression ratio of the proposed technique achieves better than the standard JPEG method. The proposed entropy encoding technique enhances the performance of the JPEG technique. Therefore, the experiment confirms that the proposed technique produces higher lossless compression than the Huffman Coding and this technique will be suitable for compression of all type of image files.

\section{REFERENCES}

[1] C. E. Shannon, "A Mathematical Theory of Communication”, in Bell System Technical Journal, vol. 27, July, October 1948, pp. 379-423,623-656.

[2] Gonzalez, R.C. and Woods, R.E., "Digital Image Processing”, 2nd ed., Pearson Education, India, 2005.

[3] Huffman, D.A., "A method for the construction of minimum-redundancy codes", Proc. Inst.Radio Eng. 40(9), pp.1098-1101, 1952.

[4] Steven Pigeon, Yoshua Bengio,"A Memory-Efficient Huffman Adaptive Coding Algorithm for Very Large Sets of Symbols", Université de Montréal, Rapport technique \#1081.

[5] Steven Pigeon, Yoshua Bengio,"A Memory-Efficient Huffman Adaptive Coding Algorithm for Very Large Sets of Symbols Revisited”, Université de Montréal, Rapport technique\#1095.

[6] R.G. Gallager,"Variation on a theme by Huffman”, IEEE. Trans. on Information Theory, IT-24(6), 1978, pp. 668-674.

[7] D.E. Knuth,”Dynamic Huffman Coding”,Journal of Algorithms, 6, 1983 pp. 163-180.

[8] C. Saravanan \& R. Ponalagusamy,'Lossless Grey-scale Image Compression using Source Symbols Reduction and Huffman Coding" International Journal of Image Processing, Vol. 3,Issue 5,pp. 246251. 
The International Journal of Multimedia \& Its Applications (IJMA) Vol.4, No.3, June 2012

[9] Jagdish H. Pujar and Lohit M. Kadlaskar, "A New Lossless Method of Image Compression and Decompression Using Huffman Coding Techniques", Journal of Theoretical and Applied Information Technology,pp. 18-23.

[10] Chiu-Yi Chen; Yu-Ting Pai; Shanq-Jang Ruan, "Low Power Huffman Coding for High Performance", Data Transmission, International Conference on Hybrid Information Technology, 2006, 1(9-11), 2006 pp.71 - 77.

[11] Lakhani, G, “Modified JPEG Huffman coding”, IEEE Transactions Image Processing, 12(2), 2003 pp. $159-169$.

[12] K. Rao, J. Hwang, “Techniques and Standards for Image, Video, and Audio Coding”, Prentice-Hall, Inc., Upper Saddle River, NJ, USA, 1996.

[13] W.B. Pennebaker, J.L. Mitchell, "JPEG Still Image Data Compression Standard," $2^{\text {nd }}$ ed., Kluwer Academic Publishers, Norwell, MA, USA, 1992.

[14] B. Sherlock, A. Nagpal, D. Monro, “A model for JPEG quantization", Proceedings of the International Symposium on Speech, Image Processing and Neural Networks (ISSIPNN-94), vol. 1, 13-16 April, IEEE Press, Piscataway, NJ, 1994.

[15] Hsien-Wen Tseng, Chin-Chen Chang, “ A Very Low Bit Rate Image Compressor Using Transformed Classified Vector Quantization”, Informatica ,29 (2005) ,pp. 335-341.

[16] Lenni Yulianti and Tati R.Mengko, "Application of Hybrid Fractal Image Compression Method for Aerial Photographs", MVA2OOO IAPR Workshop on Machine Vision Applications, Nov. 2830.2000,pp. 574-577.

[17] S.Parveen Banu, Dr.Y.Venkataramani,“An Efficient Hybrid Image Compression Scheme based on Correlation of Pixels for Storage and Transmission of Images”,International Journal of Computer Applications (0975 - 8887) Volume 18- No.3, 2011,pp. 6-9.

[18] http://nptel.iitm.ac.in/courses /Webcourse contents / IIT \% 20Kharagpur /Multimedia \% 20Processing /pdf ssg_m6116.pdf.

[19] GRGIC et al., "Performance analysis of image compression using wavelets", IEEE Trans. Indus.Elect., Vol. 48, No. 3, June 2001,pp. 682-695.

[20] Kamrul Hasan Talukder and Koichi Harada,"Haar Wavelet Based Approach for Image Compression and Quality Assessment of Compressed Image", International Journal of Applied Mathematics, 36:1, IJAM_36_1_9,2007.

[21] Rene J. van der Vleuten, Richard P.Kleihorstt, Christian Hentschel ,"Low-Complexity Scalable DCT Image Compression", 2000 IEEE.

[22] S. Smoot, L. Rowe, "Study of DCT coefficient distributions", Proceedings of the SPIE Symposium on Electronic Imaging, vol. 2657, SPIE, Bellingham, WA, 1996.

[23] D. Monro, B. Sherlock, “Optimum DCT quantization”, Proceedings of the IEEE Data Compression Conference (DCC-93), IEEE Press, Piscataway, NJ, 1993.

[24] F. Muller, "Distribution shape of two-dimensional DCT coefficients of natural images", Electron. Lett. 29 (October (22)) (1993), pp. 1935-1936.

[25] Hong, S. W. Bao, P., "Hybrid image compression model based on subband coding and edge preserving regularization", Vision, Image and Signal Processing, IEE Proceedings, Volume: 147, Issue: 1, Feb 2000,pp. 16-22. 
The International Journal of Multimedia \& Its Applications (IJMA) Vol.4, No.3, June 2012

[26] Zhe-Ming Lu, Hui Pei ,"Hybrid Image Compression Scheme Based on PVQ and DCTVQ”, IEICE Transactions on Information and Systems archive, Vol E88-D, Issue 10 ,October 2006.

[27] Charles F. Hall., “A Hybrid Image Compression Technique” CH2I 18-8/85/0000-0149 @ 1985, IEEE.

[28] Ms.Mansi Kambli1 and Ms.Shalini Bhatia,"Comparison of different Fingerprint Compression Techniques", Signal \& Image Processing: An International Journal(SIPIJ) Vol.1, No.1, September 2010, pp. 27-39.

[29] M.Mohamed Sathik , K.Senthamarai Kannan , Y.Jacob Vetha Raj, "Hybrid JPEG Compression Using Edge Based Segmentation”, Signal \& Image Processing : An International Journal(SIPIJ) Vol.2, No.1, March 2011,pp.165-176. 